\title{
Repensar el Caribe desde los Estudios Culturales: Literatura, Nación, Racialidad y Género en Donnete Francis, April Shemak y Rosamond King1
}

\begin{abstract}
Resumen
Desde los estudios literarios y culturales, estas autoras se unen a un creciente número de expertas interesadas en analizar el impacto de las dinámicas neoliberales en los aspectos étnicos, sexuales y de género en el Caribe, ya sea en el archipiélago o en sus bordes continentales. Respecto a este análisis se pueden afirmar, por lo menos, dos rasgos relevantes: el primero es la importancia otorgada a las nuevas diásporas y su efecto en la actualidad; el segundo es la necesidad de ampliar el archivo literario mediante la integración de la música, la fotografía y el performance al proceso tradicional de hermenéutica. Así, al plantearse formas más incluyentes de interpretación, la crítica busca superar lugares comunes ya desgastados por el excesivo uso y sintonizarse con las inquietudes de creadoras y creadores jóvenes respecto a las realidades de la migración y su influjo en el actual redimensionamiento político del cuerpo. En esa medida, April Shemak, Donnete Francis y Rosamond King y otras escritoras problematizan la idea de un Caribe de puro contacto y difusión.
\end{abstract}

Palabras claves

Literatura Caribeña Contemporánea, Estudios Culturales Caribeños, Diáspora, Racialización, Género, Sexualidad, Anti-romance.

\begin{abstract}
Coming from literary and cultural studies, these authors are joining a growing number of experts interested in analyzing the impact of neoliberal dynamics in ethnicity, sexuality and gender in the Caribbean, whether in the archipiélago or its continental edges. All the more, there are at least two important features that can be stated about their analysis: the first one is the importance given to

${ }^{1}$ Este escrito fue presentado inicialmente como ponencia en la "II Conferencia de Teorías y Literaturas en el Caribe y Latinoamérica", realizada en Barranquilla, departamento del Atlántico, Colombia, del 9 al 12 de septiembre de 2013, organizada por la Pontificia Universidad Javeriana y la Universidad del Atlántico.
\end{abstract}


the new diasporas and their effect on the present; the second one is the need to expand the literary archive by integrating music, photography and performance to the traditional process of hermeneutics. Thus, when considering more inclusive forms of interpretation, criticism seeks to overcome clichés worn by an excessive use and tune in on the concerns of young writers about the realities of migration and its influence on the current political resizing of the body. To that extent, April Shemak, Donnete Francis, Rosamond King and others problematize the idea of a Caribbean of pure contact and diffusion.

Keywords Contemporary Caribbean Literature, Caribbean Cultural Studies, Diaspora, Racialization, Gender, Sexuality, Anti-romance.

Creo no equivocarme al decir que este ensayo trata, a grandes rasgos, del desarrollo de un campo todavía en construcción. No obstante, para ser más exacto, debo decir que el asunto a tratar aquí son las últimas publicaciones de Donnete Francis, April Shemak y Rosamond $\mathrm{King}^{2}$, tres académicas quienes desde Norteamérica vienen desarrollando una significativa labor investigativa acerca de nuestras subjetividades históricamente subordinadas y sus conflictos y negociaciones con la hegemonía de un diseño colonial que se niega a desaparecer. Y aunque el propósito aquí no es otro que invitar a su lectura, no cabe duda de que estas conversaciones ayudan a consolidar también ese terreno inestable que algunos llamamos Estudios Culturales Caribeños. Detenernos, sin embargo, en este punto, implicaría una desviación de nuestro propósito inicial. Por eso, teniendo en cuenta lo anterior, continuaré señalando lo esencial: que estas páginas hacen un recorrido sucinto por los principales puntos de vista de estas tres autoras y sus escritos, los cuales, aparecidos en el transcurso de los últimos años, se ocupan de estudiar el impacto de la globalización en los discursos y contra discursos que históricamente han definido la región. Ellas hacen parte de un creciente número de expertas interesadas en analizar el impacto de las dinámicas neoliberales en los aspectos étnicos, sexuales y de género en el Caribe, ya sea en

${ }^{2}$ April Shemak es profesora de Literatura Postcolonial en la Sam Houston State University, ha publicado en Textual Practice y Modern Fiction Studies. Donnete Francis, por otra parte, enseña en Binghampton University, sus ensayos han aparecido en revistas tan prestigiosas como Small Axe y Black Renaissance. Rosamond King, por último, es artista y profesora, su trabajo se ha publicado en Callaloo y Woman and performance. Actualmente es profesora asociada del Brooklyn College. 
CATEDRAL TomAda: Revista de crítica literaria latinoamericana / Journal of Latin American Literary Criticism Repensar el Caribe desde los Estudios Culturales: Literatura, Nación, Racialidad y Género en Donnete Francis, April Shemak y Rosamond King

el archipiélago o en sus bordes continentales. Por su parte, con respecto a este análisis, se pueden afirmar a lo menos dos rasgos relevantes, que dichos brevemente, son los siguientes: el primero es la importancia otorgada a las nuevas diásporas y su efecto en la actualidad; el segundo es la necesidad de ampliar el $\operatorname{archivo}^{3}$ mediante la integración de la música, la fotografía y el performance al proceso tradicional de hermenéutica. Así, al plantearse formas más incluyentes de interpretación, la crítica busca superar lugares comunes ya desgastados por el uso excesivo y sintonizarse con las inquietudes de creadoras y creadores jóvenes respecto a las realidades de la migración y $\mathrm{su}$ influjo en el actual redimensionamiento político del cuerpo.

Es en esa medida que en su Asylum speakers: Caribbean Refugees and Testimonial Discourse, aparecido en el 2011, April Shemak problematiza la idea de un Caribe de puro contacto y difusión ${ }^{4}$. Basta decir, para resumirlo, que el libro gira en torno a la problemática representación del refugiado por parte del gobierno y la sociedad norteamericana, por un lado, y de quienes emigran en condiciones de marginalidad y pobreza, por el otro. En un principio, la autora contrasta las fotografías de refugiados tomadas por la guardia costera estadounidense con una narrativa compuesta por escritoras descendientes de emigrados caribeños que originalmente publican en y para el mercado anglo. Lo que interesa en este caso es ver en que formas el discurso oficial estadounidense ha representado el desplazamiento de comunidades discriminadas en sus propios ámbitos nacionales a través de un discurso abiertamente racista, en el cual se observa un subtexto donde los inmigrantes se erigen por igual en víctimas y desechos indeseables de una tierra salvaje opuesta totalmente al "sueño

${ }^{3}$ El "Archivo", sostiene González Echavarría en Myth and Archive: a Theory of Latin American Narrative. (1990), es esa novela que al marcar el punto de inicio de una tradición, tiene un efecto modelador sobre otros textos posteriores, mientras que al mismo tiempo constituyen un reservorio de las posibilidades estéticas de su tiempo. Aquí se busca extender esa noción de lo escritural hacia una semiótica de lo visual y del performance.

${ }^{4}$ En español traduce Los Portavoces del asilo: los refugiados caribeños y el discurso testimonial. (New York: Fordham University Press, 2011), 310 pp. Hasta la elaboración de este escrito no había traducción. 
americano". Como se dijo antes, a este discurso oficial se contrapone una tradición literaria propia, en la cual el nombre de Edwige Dandicat resulta especialmente significativo. Solo digamos al respecto que Dandicat ha escrito ampliamente sobre la difícil recepción de la comunidad haitiana a la que pertenece por parte de la "american society". En Brother, I'm dying (2008), por mencionar un ejemplo, la escritora reconstruye el confinamiento, maltrato y absurda muerte de un familiar suyo en un centro de detención de Miami tras su escape del régimen infame de Duvalier. Sin embargo, cabe decir que estas realidades no se limitan solo a la experiencia de Haití. En lo referente a la literatura y la academia norteamericana que se ocupa del Caribe, existen otras obras y flujos migratorios mediante los cuales re-pensar las interconexiones entre sexualidad, género, raza y ciudadanía en la región.

Una muestra de esta afirmación sería Donette Francis con Fictions of Feminine Citizenship: Sexuality and the Nation in Contemporary Caribbean Literature $(2010)^{5}$, en la cual ni siquiera la migración vista como escape de la pobreza y opresión doméstica se constituye en un nicho plenamente seguro de inscripción, sobretodo en una época de profundos conflictos y contradicciones que se hacen mucho más palpables si nos referimos a la dimensiones culturales del cuerpo. Allí habría que señalar, haciendo un paréntesis, las similitudes entre Francis y Shemak en la forma como ambas leen el pasado y presente de dicha corporeidad, hecho que obedece al uso común de metodologías procedentes de la teoría postcolonial, teoría queer, historiografía, examen de archivo y fotografía. Perspectivas todas que (no ampliaré más por falta de espacio), constituyen el eje metodológico y teórico de los así llamados Estudios Culturales Caribeños. Regresando al cuerpo, no obstante, a su inscripción social y a su realidad física atravesada por el discurso, las dos comparten la opinión de que muchas mujeres y hombres pueden llegar a ser mucho más susceptibles al abuso y la explotación en

5 Ficciones de la ciudadanía femenina: Sexualidad y Nación en la Literatura Caribeña contemporánea. (New York: Palgrave Macmillan, 2010), 191 pp. Tampoco de este libro conozco traducción hasta el momento. 
CATEDRAL TomAda: Revista de crítica literaria latinoamericana / Journal of Latin American Literary Criticism Repensar el Caribe desde los Estudios Culturales: Literatura, Nación, Racialidad y Género en Donnete Francis, April Shemak y Rosamond King

los reflujos de la emigración que en sus propias naciones de origen. Para demostrar como esto se refleja en la narrativa en cuestión, el trabajo de Francis se basa en novelas que además de ser muy recientes provienen de diferentes paisajes lingüísticos y nacionales, lo que de paso confirma que las interconexiones que ya se habían mencionado no se expresan siempre en un perfil único y universalmente válido. Entre estas novelas, que mencionaré en desorden, se encuentran The pagoda de Patricia Powell, Song of the wáter saints de Nelly Rosario, Breath, eyes, memory de Edwige Dandicat, Bruised hibiscus de Elizabeth Nuñez y Soledad de Angie Cruz ${ }^{6}$. Y aunque no creo necesario ahondar demasiado en esta cuestión, digamos que este enfoque evidencia la tendencia cada vez más marcada del trabajo académico caribeño contemporáneo hacia lo transnacional. Empero, en lo que nos ocupa, apuntemos que la razón de este rastreo por partida quíntuple es mostrar la manera oscilante y compleja en que nuestras geografías transnacionales han sido sujetas a un ordenamiento bio-político colonial, el cual se muestra incapaz de asumir realmente la diferencia mientras que al mismo tiempo diluye sus prejuicios en la alcoba. Esta dualidad manifiesta de lo sexual es uno de los ejes problemáticos más interesantes de la autora, quien nos ofrece este interesante volumen sobre cómo de las plantaciones y haciendas hasta los resorts internacionales el etnocentrismo en el poder se ha encontrado con la otredad en el micro-espacio del dormitorio. Paradójicamente, aunque pareciera que en este encuentro se deslegitimasen verdades raciales y culturales fijas y las conciencias se abrieran a nuevas posibilidades, la realidad por si sola se encarga de mostrar estas uniones en su carácter insuficiente: la situación marca un contraste por cuanto estos encuentros van de la mano, por un lado, de la fusión y aceptación producida por lo íntimo y, por el otro, de la creación de esencialismos que a la postre han desembocado en nichos sociales extensamente socorridos, los cuales, en virtud del pensamiento unívoco que nos ha sido impuesto, han sido

${ }^{6}$ Algunas de estas autoras también son abordadas por April Shemak, aunque su perspectiva es diferente. Además de este grupo de escritoras, Shemak agrega nombres de jóvenes investigadoras en Estudios Culturales, caso de Omise Eke Natasha Tinsley, por ejemplo. 
naturalizados y erigidos en entidades auto-evidentes. Estos nichos son la plataforma desde donde se justifican la xenofobia y la discriminación a la que se refiere April Shemak en su obra. La relación aquí reside en el hecho de que, partiendo del plano personal para aterrizar en el político, se entretejen vínculos entre la falsa integración y el racismo implícito en la versión clásica del mestizaje y el doblez de un discurso institucional que enarbola en público una integración que tácitamente rechaza.

Más aún, si hablamos de esencialismo, tenemos que uno de sus rasgos más perniciosos es la "commodificación" sexual de ciertos tipos humanos en el mercado internacional, una problemática muy documentada por la academia y ampliamente explotada por los medios. El libro toca ese tema y no es el único. Lo valioso en este caso es la devolución que Francis hace de la propia individualidad a los individuos explotados por esta maquinaria, puesto que su reificación dentro un régimen de abuso no implica atribuirles a estos individuos una inercia total. Francis parte de que la prostitución del caribeño, ya sea en el área o fuera de ella, a pesar de que en muchos casos equivalga a una esclavitud moderna, ciertamente se erige en una forma de supervivencia donde las trabajadoras y trabajadores venden un servicio altamente cotizado con el que ganan un importante empoderamiento económico que, en mayor o menor medida, repercute en su bienestar y el de sus familias. De esta manera ambigua, sopesando los pros y contras, Donnete Francis propone una nueva perspectiva para entender las peculiares dinámicas de negociación de estos sujetos con el sistema que los vulnera, sin desconocer, por supuesto, las realidades del poder inscritas en sus personas. Visto a través del lente de Francis, este uso del deseo para equilibrar la balanza hace parte de un proyecto más amplio de “de-colonialidad”. Más amplio porque esta necesidad de liberación no se aplica solo a las circunstancias que rodean a la imposición colonial o la transacción monetaria sino a toda manifestación erótica, pues en la migración y en cada uno de nuestros sistemas nacionales estas identidades, tan plurales en esta parte del mundo como en cualquier otro lugar, han estado siempre mediadas, proscritas y prescritas por la 
CATEDRAL TomAda: Revista de crítica literaria latinoamericana / Journal of Latin American Literary Criticism Repensar el Caribe desde los Estudios Culturales: Literatura, Nación, Racialidad y Género en Donnete Francis, April Shemak y Rosamond King

norma heterosexual y el privilegio tácito del varón de ascendencia europea, patrón dominante dentro de esa especie de cadena evolutiva que ha informado dichos sistemas desde sus inicios. Lo curioso sobre Shemak y Francis es que su investigación evidencia como ahora que se le piensa superado, ese paradigma muestra su tenaz y perniciosa persistencia, modelando nuestro patrimonio cultural y nuestras maneras de entenderlo. Por esa razón hacer resistencia, "decolonializarse" nos dicen ambas, es combatir estas mediaciones dentro de lo vivencial, desde la literatura y caso concreto del campo intelectual, implica un ataque directo a corrientes de pensamiento tradicionalmente aliadas de la dominación. En ese proceso, ambas pasan de lamentar la indefensión de las y los individuos ante una geopolítica inicua a ver como ellos, sujetos de derecho, intentan tomar las riendas de su propia existencia en cuanto seres sociales. En el caso concreto de las narradoras contemporáneas de las diásporas caribes ${ }^{7}$, según lo piensan Francis y Shemak, esto se logra a través de variadas modalidades narrativas, de las cuales nos interesa la que Francis denomina como Antiromance $^{8}$. Sin excepción alguna, las novelas mencionadas constituyen ejemplos

${ }^{7}$ Diáspora se refiere aquí a los flujos migratorios contemporáneos de Caribeños y caribeñas hacia el primer mundo.

${ }^{8}$ Debemos recordar que en la tradición literaria de habla inglesa "Romance" designa una tipología narrativa cuyo foco principal está constituido por las relaciones sentimentales. Orgullo y prejuicio de Jane Austen sería un ejemplo clásico de esto. Los "Romances" están marcados por un tono emocionalmente exaltado y la omnipresencia de los finales felices. En el plano moral, este tipo de textos se caracteriza por la exaltación de los valores tradicionales y el castigo simbólico de las conductas no convencionales. A lo dicho se aúna el ideal del amor incondicional: los personajes del "Romance" clásico, lánguidas señoritas y galanes decididos, se caracterizan por la búsqueda constante de una felicidad doméstica que por regla general es alcanzada con el matrimonio que se da hacia el final. El "Romance", sin duda, es el precedente ingles del "Folletín". A su vez, Cabrera Infante afirma, en la mesa redonda titulada "El folletín por entregas y el serial", realizada en 1982 en el marco del Festival de cine de San Sebastián, que los orígenes de este tipo de escritos deben buscarse en las novelas medievales de Caballería. Es contraponiéndose a esta tradición del "Romance" y de la novela rosa "folletinesca", a su cosmovisión patriarcal cortesana y a su peculiar modo de estereotipar la sexualidad humana y las relaciones de género, que surge el Antiromance del que aquí se habla. Más aún, insisto en que el Anti-romance es una categoría acuñada por Francis para entender una parte de la narrativa de la zona. En esa medida, seguimos hablando de los rasgos de ciertas novelas actuales, entre las cuales están obviamente las autoras previamente citadas. En lo que yo conozco, me atrevería a agregar los nombres de Cristina García, Mayra Santos Febres y Antonia Palacios, aunque seguramente debe haber muchísimas otras. 
claros de anti-romances. No obstante, antes de definir porque lo son, hay que aclarar que la visión melodramática del "Romance" ha sido el modo tradicional en que la cultura de masas ha entendido los roles de género desde hace por lo menos unos doscientos años. A pesar de esta antigüedad e influencia, para ella es imposible obviar la irrealidad de esta representación en un territorio signado por el auténtico silenciamiento, indefensión e impotencia que todavía padecen muchas personas, tanto hombres como mujeres.

Lo que nos dice lo anterior es que la validación del acallamiento, abuso e imposición domestica por parte del "Romance" contribuye no sólo a la vulneración de la subjetividad femenina por parte del poder patriarcal, sino también a la frustración causada por la segregación dentro de un esquema masculino jerarquizado, racializado y fragmentado en clases. En contra de la supuesta calma del melodrama, el Antiromance da voz a aquellos que han sido reprimidos por una "normalidad" cuya felicidad es puramente aparente. Es más, en lo estrictamente sexual, algunos de estos antiromances se convierten en lo que la investigadora entiende por Archivos de intimidad ${ }^{9}$, es decir, en documentos que revisan su mediación en la construcción discursiva de las sociedades y, por ende, reflexionan sobre como ciertas nociones de ciudadanía se reflejan en las relaciones íntimas. Lo que se quiere decir es que pese al camino recorrido, la pasividad de muchas mujeres y su exclusión de la res pública corre paralela a su abnegación y renuncia en el terreno de lo erótico y a su cancelación de ciertas ambiciones y expectativas en sus vidas. En el mismo extremo, cualquier expresión de homosexualismo u otra manifestación de la diferencia se encuentra sometida al bloqueo y la censura, por tanto hoy es bien sabido que en gran parte

${ }^{9}$ Al principio de este comentario ya se había señalado la necesidad de expandir lo que se pensaba del archivo según lo define González Echavarría. Hablar de archivos de intimidad es revisar a) la exposición y reificación de la mujer dentro de los regímenes visuales patriarcales (Nelly Rosario), b) los traumas del despotismo (caso de Edwige Dandicat), y c) el privilegio eurocéntrico que justifica el sometimiento de pueblos violados y arrasados, atados a una voluntad legitimada en su propia brutalidad y sentido de superioridad. 
CATEDRAL TomAda: Revista de crítica literaria latinoamericana / Journal of Latin American Literary Criticism Repensar el Caribe desde los Estudios Culturales: Literatura, Nación, Racialidad y Género en Donnete Francis, April Shemak y Rosamond King

de nuestros países estas alternativas pasan de ser estigmatizadas en el dominio de lo privado a su negación en la esfera gubernamental ${ }^{10}$.

Los archivos de intimidad, por el contrario, proponen un reencuentro con la sensualidad en todas sus formas, lo que es una declaración política porque la aceptación de la otredad se conjuga con una voluntad de ampliar los ámbitos civiles cerrados por un pensamiento excluyente, entendiendo lo marginado no solo como aquello que traspasa las normas convencionales de género, sino lo que asimismo desregula las uniones interraciales. Ahora bien, de la idea de los Archivos de intimidad pasemos a otro punto importante del libro de Francis, más exactamente al modo en que este se inserta de manera más directa en el debate feminista, lo que curiosamente se logra mediante la exploración de una faceta pictórica. A través del examen de la portada, constituida por la obra de Roshini Kempado "Virtual exiles: from the edge", se reconstruye el modo en que la escuela, la familia y el ejército han modelado la acción y la conciencia de las mujeres en los ámbitos nacionales e internacionales del Gran caribe. Sin embargo hay que señalar, ante todo, que lo importante de este análisis no son las conclusiones, por lo demás esclarecedoras, que su lectura nos ofrece, sino su interesante uso de textualidades alternas para hacer una interpretación abierta y multinivel de un hecho social. Así, partiendo de un signo visual obviado hasta hace muy poco por la semiótica literaria se busca identificar los significantes arbitrarios surgidos en el seno de dicha institucionalidad, de-construyendo en esa forma sus estrategias específicas de control y explotación. Sin embargo, Fictions of Feminine Citizenship: Sexuality and the Nation in Contemporary Caribbean

\footnotetext{
${ }^{10}$ Nadie ignora las resistencias institucionales por las que atraviesa el movimiento LGBT en el país. Leo al momento de escribir este comentario la noticia de que un juez ha anulado el segundo matrimonio homosexual realizado en Colombia como respuesta una demanda interpuesta por una Fundación por los valores tradicionales. La nota aparece en El Universal de Cartagena del día 19 de Octubre de 2013

${ }^{11}$ De origen Coolie, Roshini Kempadoo es una fotógrafa y artista visual ampliamente reconocida, nació en Gran Bretaña pero ha vivido la mayor parte de su vida entre Jamaica, Trinidad, Barbados y Guyana. Actualmente enseña artes visuales en la Universidad de East London. Virtual Exiles (2000) es una conocida serie fotográfica basada en una estadía de dos años en Guyana a finales de los noventa.
} 
Literature (2010) no se trata solamente de reafirmar una problemática o una especie de capacidad femenina de agencia, sino de reinterpretar cómo dicha agencia se entrecruza y crea lazos de irrecusable solidaridad con aquellos discursos que hoy en día giran en torno a los todavía inagotados debates sobre racialidad, nación, lenguaje y saberes autóctonos en nuestra social, política y lingüísticamente fragmentada geografía.

Finalmente, otra temática importante dentro de los Estudios Culturales es la discusión sobre la presunta solidez de la diada masculinidad - femineidad en la región. De hecho, visto en perspectiva, y tal y como se afirmó antes, podría pensarse en los discursos de la "Creolización y el mestizaje" 12 como parte del emplazamiento de la norma patriarcal en la comprensión intelectual del Caribe. Un hecho que cuestionaría este paradigma seria, pese a lo dicho, la existencia de espacios en los que los rasgos sexuales de la personalidad resultan ser arquitecturas movedizas, ambiguas o indefinidas. Al respecto, Rosamond King ha escrito muy recientemente un ensayo ${ }^{13}$ sobre una subjetividad subordinada que para criticar al sistema se posiciona temporalmente en una pluralidad no heteronormativa que da cabida a todas las formas del deseo tradicionalmente proscritas. Lo que le interesa a Smith es ver cómo a través de ciertas prácticas lúdicas y sexuales pasajeras se pueden transformar, así sea por poco tiempo, códigos impuestos de comportamiento y mostrar la inconformidad por una inscripción identitaria arbitraria. El performance y la música son importantes en esta transformación porque a través suyo el poder transformador de lo lúdico y lubrico parece proyectarse con más fuerza, algo que puede notarse en ese hecho a la vez tan tradicional y transitoriamente subversivo que viene a ser el carnaval. Dicho con propiedad, Smith no agrega nada nuevo en el campo de la teoría.

${ }^{12}$ Valga la aclaración de que aquí hablo de los discursos sobre lo Creole (por ejemplo el de Brathwaite) que piensan la creolidad en el ámbito de la plantación y que en ningún momento me refiero a la Creolidad de Confiant, Chamoiseau y Bernabe. Por otro lado, un ejemplo del concepto de mestizaje es la idea propuesta por los pensadores de la revolución (Guillén por ejemplo) de que el mulato es el elemento definidor de la identidad biológica y cultural cubana.

${ }^{13}$ King, Rosamond (2011). "New citizens, New sexualities: Nineteenth century Jamettes", en Sex and the Citizen: Interrogating the Caribbean. Faith Smith, compiladora. Charlottesville and London: University of Virginia Press. 
CATEDRAL TomAda: Revista de crítica literaria latinoamericana / Journal of Latin American Literary Criticism Repensar el Caribe desde los Estudios Culturales: Literatura, Nación, Racialidad y Género en Donnete Francis, April Shemak y Rosamond King

Ciertamente, la idea de que el travestismo es, entre otras muchas cosas, una trasgresión de las jerarquías, es harto común. Aun así, lo que su trabajo tiene de peculiar y útil es la lectura de esa transgresión en el circuito específico de los "Jamettes" de Trinidad y la semiótica implícita en su indumentaria y gestualidad $^{14}$. King sostiene que si bien su puesta en escena implica una desaparición solo pasajera de su subordinación, los disfraces de los "Jamettes" se convierten en un contra-discurso mediante el cual los habitantes de la isla desafían la supremacía de un legado victoriano cargado de estereotipos, excesivamente rígido y moralista. Curiosamente, ha sido mediante su supuesta "vulgaridad" festiva, tan ofensiva a la plantocracia decimonónica, que la gente triniteña, los hombres y mujeres humildes que han tomado parte en esta celebración, articulan un sentido de su valor personal y el de sus propios saberes y cultura. Ya sea apelando al color, la música y el ruido, parodiando la imagen de la dama, satirizando el atuendo y exagerando los ademanes del caballero inglés, oponiéndole una versión estridente y estrafalaria de sí mismo, intercambiando, desdibujando o modificando lo que significa ser hombre o mujer, travistiéndose y actuando en maneras deliberadamente insinuantes, permitiendo al esclavo vestirse y comportarse igual que el amo, usando indumentarias tradicionales o abriendo espacios de intimidad donde, aunque sea por poco tiempo, se acepte la consumación de todos los apetitos sin importar cuales sean, estos segmentos de la sociedad colonial han desnaturalizado las posiciones sexuales, raciales y sociales, mostrándolas en su carácter de construcción y colocando en tela de juicio los valores establecidos por la dominación occidental.

Por esta razón, más allá de lo folclórico, King considera que la atención puesta a los "Jamettes" permite a la comunidad de estudiosos ver como a través

\footnotetext{
14“"Jamette" es un vocablo creole que originalmente se usaba para designar a las personas "poco respetables" dentro de los parámetros victorianos. Haciendo una asociación con su supuesto carácter licencioso, el término "Jamette carnival" fue usado por los plantadores británicos para describir los carnavales de negros. En esa medida, "Jamettes" vendrían a ser todos los hombres y mujeres que participaban y participan en el carnaval. Aún hoy en día los llamativos, descubiertos y coloridos disfraces de esta festividad siguen siendo considerados controversiales en virtud de su abierto travestismo y la marcada corporalidad de su performance.
} 
del uso ideológico del enmascaramiento y la corporalidad, los creoles negros de esta parte del archipiélago han construido modos únicos de entender una libertad recién adquirida e incompleta, necesariamente diferente de la articulada por los segmentos europeos, minoritarios y terratenientes. Al igual que en los trabajos anteriores, lo que se aporta, aparte de los resultados de la investigación, debe ubicarse en el terreno del método: sin duda King nos permite conocer más de ese evento particular; pero también esboza la posibilidad de establecer contrastes entre sus interpretaciones y los rasgos carnavalescos característicos de contextos diferentes, sobre todo a nivel de las prácticas contra-discursivas. Lo que se le puede preguntar en este caso a la teoría del performance es si los vasos comunicantes entre lo festivo y lo cotidiano se manifiestan de manera diferente dependiendo del espacio que se aborde, o si al hablar de prácticas de resistencia en toda la cuenca, los procesos a través de los cuales estas prácticas mantienen o reescriben su sentido original obedecen a unos patrones similares, marcados por una historia común. Al lado del inmediatismo que nos obligaría a decir que sí, Rosamond King deja abierto el espacio para una reflexión más profunda al respecto. Así las cosas y para no extendernos más, digamos en síntesis que la flexibilidad con que las tres autoras afrontan sus respectivos objetos de estudio, junto con el nuevo espectro de temas a abordar, abre un camino hacia nuevas formas de pensar la investigación en lo que podría pensarse como una segunda generación de Estudios Culturales Caribeños.

Considero que de esta flexibilidad y de su capacidad de repensarse a sí mismos y sus objetivos hacia el futuro depende su consolidación final o no como campo intelectual. Este acto de cuestionar la fijeza de los propios presupuestos, tarea por lo demás ardua, implica abogar por una perspectiva transdisciplinar abierta a nuevas formas de entender practicas complejas y procesos aun inacabados. Este es en propiedad el aporte de estas y otras investigadoras a los diálogos que los Estudios Culturales entablan con lo social, y es así como ellas logran ampliar también el horizonte de los estudios literarios. No debe pensarse en esta conclusión como la promoción de una panacea; los Estudios Culturales 
tienen un posicionamiento específico en el tiempo y el espacio y solo desde allí contribuyen a entender el mundo creativo de nuestros pueblos. Este posicionamiento, cuyo grueso de publicación es incuestionablemente angloparlante, forjado mayormente en los centros universitarios metropolitanos y por ello contradictorio y difícil, no se constituye ni pretende constituirse en una vía única, sino que busca complementar otros focos investigativos mientras se nutre a la vez de ellos. Estos focos, por su parte, se basan en una creciente interconexión que expresa un afán de conocernos y reconocernos más allá de las grandes diferencias que nos separan, que en realidad son tan fuertes como los lazos que nos unen. Por eso a través de un proceso cada vez más abarcador, poliglota y plurinacional, los Estudios Culturales y otras inter-disciplinas se identifican e inscriben plenamente en ese deseo, proponiendo de paso nuevas perspectivas para entender esas redes particulares de poder que actúan sobre nuestros cuerpos y conciencias y en esa medida sobre nuestras representaciones, rehusándose al estereotipo y problematizando las ideas cómodas mediante las cuales se piensa el Caribe. No se entienda esto como un ataque a nuestra innegable relación, se trata de señalar las dificultades que persisten en ese proceso paulatino de integración para superarlas desde lo que nos compete como docentes e investigadores. Justamente para cumplir con esas expectaciones es que Francis y las demás nos sugieren ir más allá de la letra y del vasto arsenal de la erudición tradicional, explorando las significaciones que lo visual y gestual-corporal vehiculan y convierten en nuestro propio testimonio.

\section{Bibliografía}

Dandicat, Edwige. Brother, I'm dying. New York: Vintage Books, 2008.

Cabrera Infante, Guillermo. "Dossier: El folletín por entregas y el serial mesa redonda”, En: Análisi, Núm. 9, 1984, 1984, 143-166. 
Francis, Donette. Fictions of Feminine Citizenship: Sexuality and the Nation in Contemporary Caribbean Literature. New York: Palgrave Macmillan, 2010 .

González Echevarría, Roberto. Myth and Archive: a Theory of Latin American Narrative. New York: Fordham University Press, 1990.

Kempadoo, Roshini. Virtual exiles, 2000. Available at:

http://www.roshinikempadoo.co.uk/gallery_22559 (15 Sept. 2013).

King, Rosamond. "New citizens, New sexualities: Nineteenth century Jamettes", En Smith, Faith. (Comp.) Sex and the Citizen: Interrogating the Caribbean. Charlottesville and London: University of Virginia Press, 2011.

Shemak, April. Asylum Speakers: Caribbean Refugees and Testimonial Discourse. New York: Fordham University Press, 2011. 